Supporting information for:

\title{
Synthesis of novel bio-lubricants from waste cooking oil and cyclic oxygenates through an integrated catalytic process
}

\author{
ACS Sustainable Chemistry and Engineering \\ Hossein Jahromi ${ }^{1,2}$, Sushil Adhikari ${ }^{1,2}$, Poulami Roy ${ }^{1}$, Madison Shelley ${ }^{1}$, Ehsan Hassani ${ }^{3}$, Tae- \\ Sik $\mathrm{Oh}^{3}$ \\ ${ }^{1}$ Biosystems Engineering Department, 200 Corley Building, Auburn University, Auburn, AL 36849, United States \\ ${ }^{2}$ Center for Bioenergy and Bioproducts, 519 Devall Drive, Auburn University, Auburn, AL 36849, United States \\ ${ }^{3}$ Department of Chemical Engineering, Auburn University, Auburn, Alabama 36849, USA
}

\section{Table of contents}

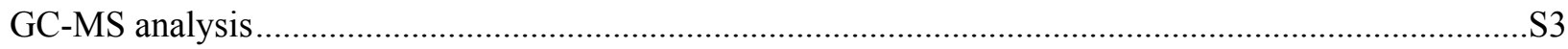

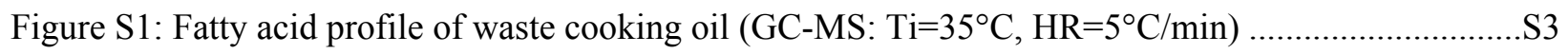

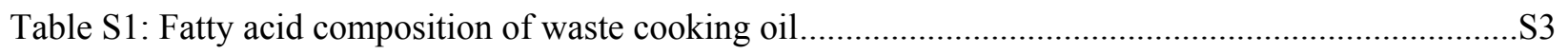

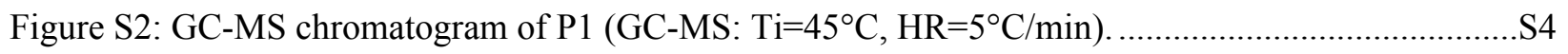

Table S2: chemical structures of P1 identified using GC-MS..............................................................S4

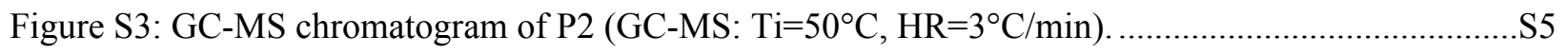

Table S3: chemical structures of P2 identified using GC-MS...............................................................S5

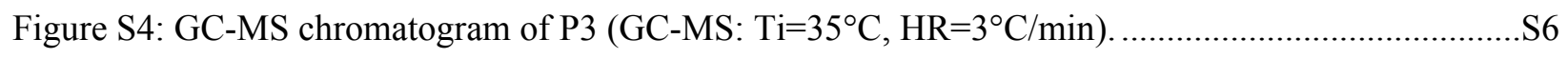

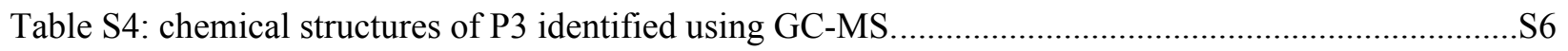

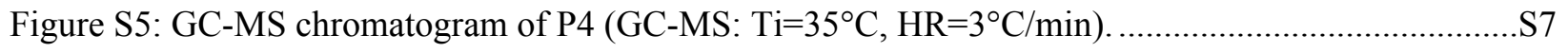

Table S5: chemical structures of $\mathrm{P} 4$ identified using GC-MS................................................................ 7

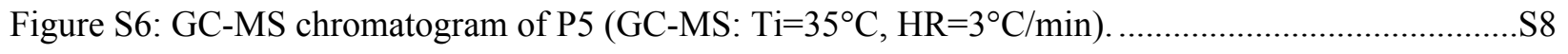

Table S6: chemical structures of P5 identified using GC-MS........................................................... 8

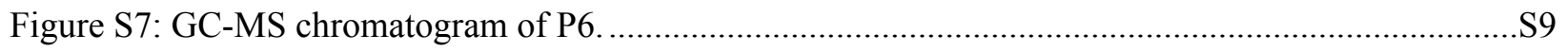

Table S7: chemical structures of P6 identified using GC-MS........................................................... 9

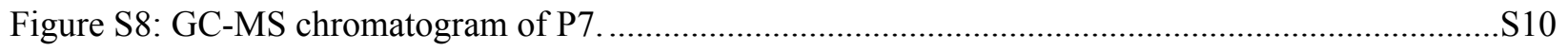

Table S8: chemical structures of P7 identified using GC-MS.............................................................. 10

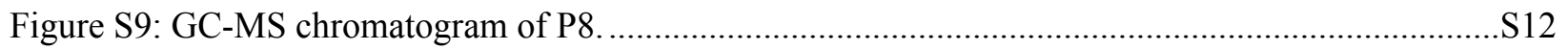

Table S9: chemical structures of P7 identified using GC-MS............................................................. 12

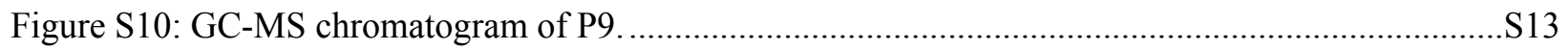

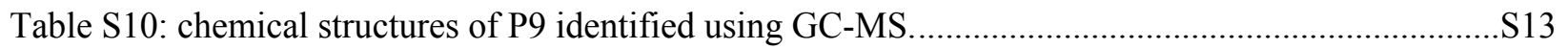

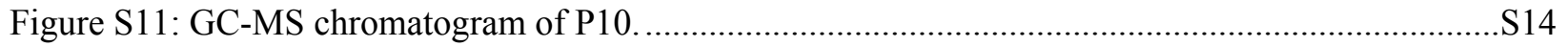

Table S11: chemical structures of P10 identified using GC-MS............................................................ 
Figure S12: GC-MS chromatogram of $\mathrm{P} 11$ (GC-MS: $\mathrm{Ti}=35^{\circ} \mathrm{C}, \mathrm{HR}=3^{\circ} \mathrm{C} / \mathrm{min}$ ). S15

Table S12: chemical structures of P11 identified using GC-MS S15

Figure S13: GC-MS chromatogram of $\mathrm{P} 12$ (GC-MS: $\mathrm{Ti}=40^{\circ} \mathrm{C}, \mathrm{HR}=5^{\circ} \mathrm{C} / \mathrm{min}$ ). S16

Table S13: chemical structures of P12 identified using GC-MS S16

Figure S14: GC-MS chromatogram of $\mathrm{P} 13$ (GC-MS: $\mathrm{Ti}=40^{\circ} \mathrm{C}, \mathrm{HR}=5^{\circ} \mathrm{C} / \mathrm{min}$ ). S 17

Table S14: chemical structures of P13 identified using GC-MS ..S17

Figure S15: GC-MS chromatogram of P14. ..S19

Table S15: chemical structures of P14 identified using GC-MS. .S19

Figure S16: GC-MS chromatogram of P15. ..S20

Table S16: chemical structures of P15 identified using GC-MS. S20

Figure S17: GC-MS chromatogram of P16. .S21

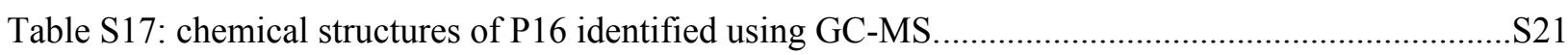

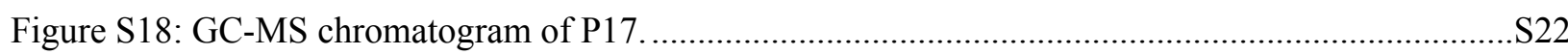

Table S18: chemical structures of $\mathrm{P} 17$ identified using GC-MS (GC-MS: $\left.\mathrm{Ti}=60^{\circ} \mathrm{C}, \mathrm{HR}=5^{\circ} \mathrm{C} / \mathrm{min}\right) \ldots \ldots . \mathrm{S} 22$

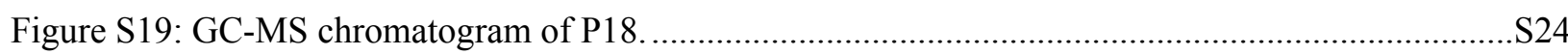

Table S19: chemical structures of $\mathrm{P} 18$ identified using GC-MS (GC-MS: $\left.\mathrm{Ti}=60^{\circ} \mathrm{C}, \mathrm{HR}=5^{\circ} \mathrm{C} / \mathrm{min}\right) \ldots \ldots . \mathrm{S} 24$

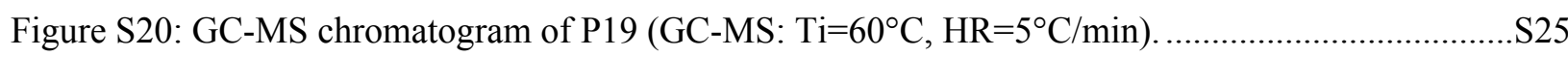

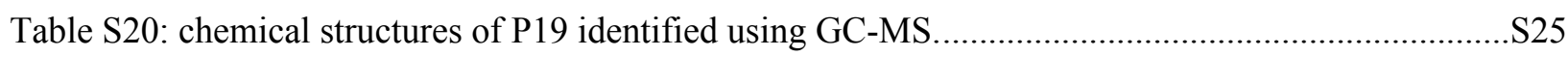

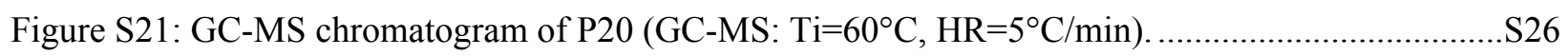

Table S21: chemical structures of P20 identified using GC-MS .S26

Number of pages: 26

Number of Figures: 21

Number of Tables: 21 


\section{GC-MS analysis}

The chemical composition of bio-lubricants was analyzed using an Agilent Technologies 7890A Gas Chromatograph (GC) System outfitted with a 7683B Series Injector and 5975C Inert Mass Selective Detector (MSD) with Triple-Axis Detector. The GC-MS was equipped with $30 \mathrm{~m}$ x 250 $\mu \mathrm{m} \times 0.25 \mu \mathrm{m}$ DB-1701 Column. An estimated $20 \mathrm{mg}$ of each sample into a clean vial and diluting each sample with dichloromethane (DCM) until each diluted sample contained nearly $2 \mathrm{wt}$ \% BL. The filled vial was then loaded into an auto sampler and injected using a $10 \mu \mathrm{L}$ syringe into the GC System. The GC oven was programmed to heat to an initial temperature of $\mathrm{Ti}=50^{\circ} \mathrm{C}$ and hold for $2 \mathrm{~min}$ before being heated at a heating rate of $\mathrm{HR}=5^{\circ} \mathrm{C} / \mathrm{min}$ to a final temperature of $280^{\circ} \mathrm{C}$ and holding time of $15 \mathrm{~min}$, unless specified otherwise.

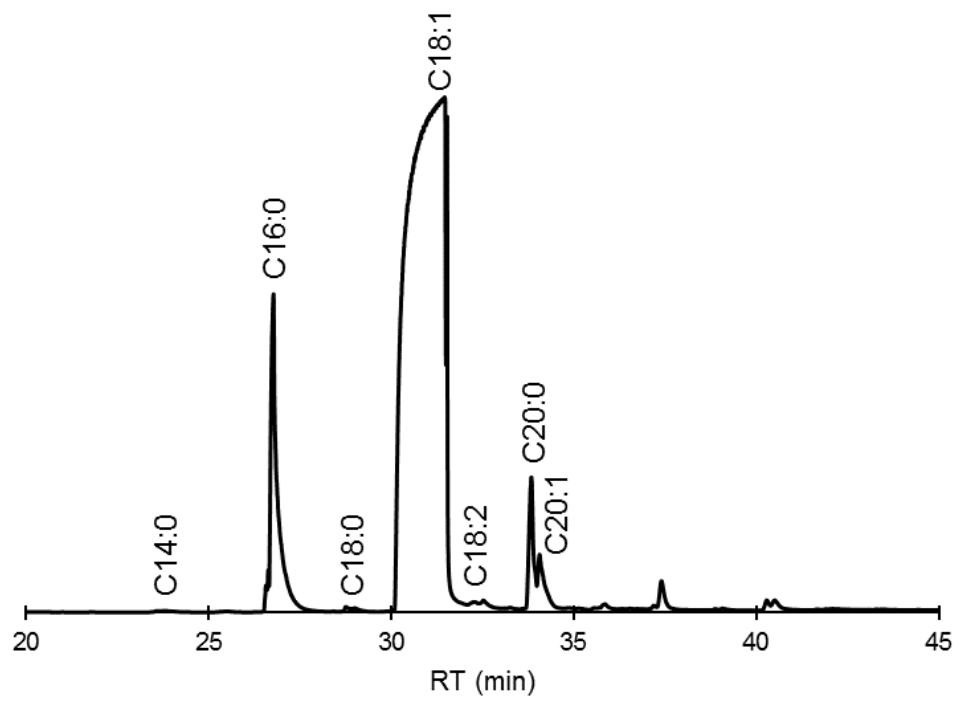

Figure $\mathrm{S} 1$ : Fatty acid profile of waste cooking oil (GC-MS: $\left.\mathrm{Ti}=35^{\circ} \mathrm{C}, \mathrm{HR}=5^{\circ} \mathrm{C} / \mathrm{min}\right)$.

Table S1: Fatty acid composition of waste cooking oil.

\begin{tabular}{lll}
\hline Type of fatty acid & Carbon chain & Composition (wt.\%) \\
\hline Myristic & C14:0 & 0.9 \\
Palmitic & C16:0 & 18.6 \\
Stearic & C18:0 & 1.7 \\
Oleic & C18:1 & 63.2 \\
Linoleic & C18:2 & 2.5 \\
Arachidic & C20:0 & 7.2 \\
Eicosenoic & C20:1 & 3.1 \\
Others & & 2.8 \\
\hline
\end{tabular}




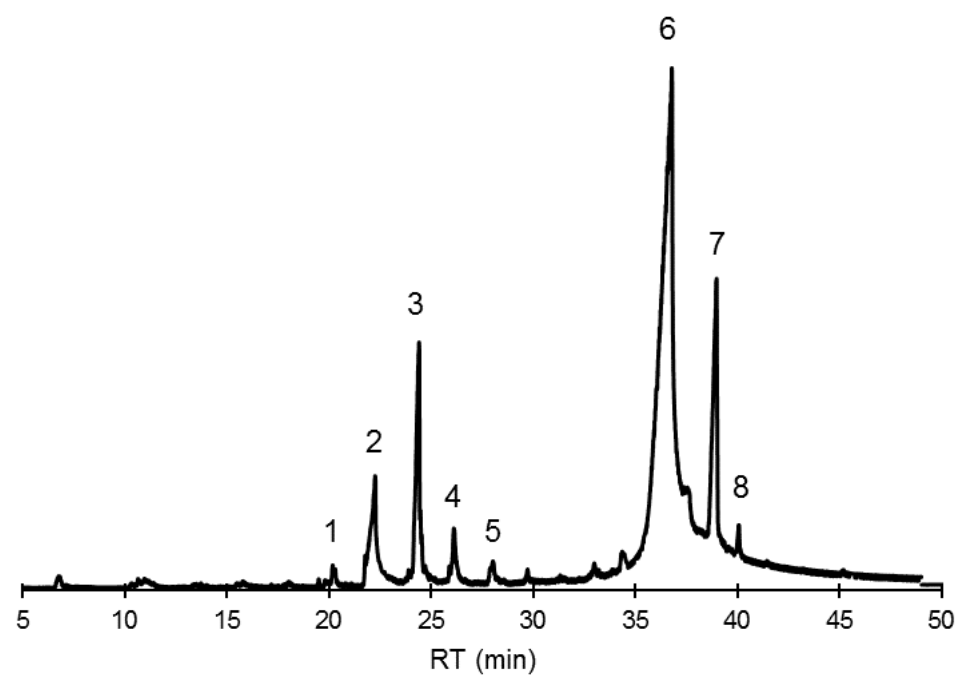

Figure $\mathrm{S} 2$ : GC-MS chromatogram of $\mathrm{P} 1\left(\mathrm{GC}-\mathrm{MS}\right.$ : $\left.\mathrm{Ti}=45^{\circ} \mathrm{C}, \mathrm{HR}=5^{\circ} \mathrm{C} / \mathrm{min}\right)$.

Table S2: chemical structures of P1 identified using GC-MS.

Peak




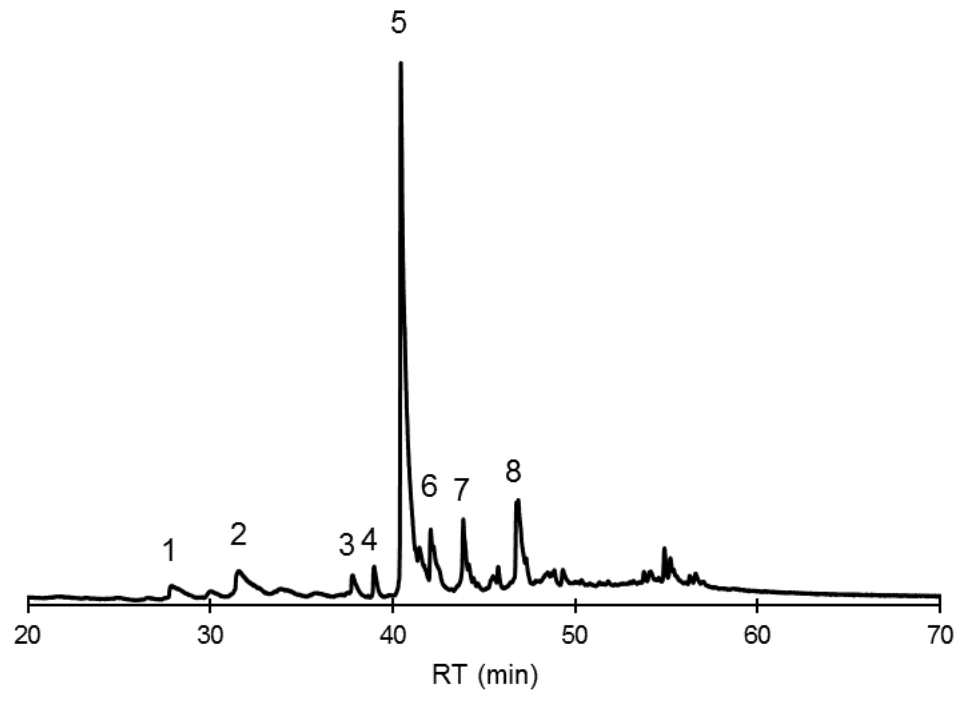

Figure S3: GC-MS chromatogram of $\mathrm{P} 2\left(\mathrm{GC}-\mathrm{MS}\right.$ : $\left.\mathrm{Ti}=50^{\circ} \mathrm{C}, \mathrm{HR}=3^{\circ} \mathrm{C} / \mathrm{min}\right)$.

Table S3: chemical structures of P2 identified using GC-MS.

Pumber




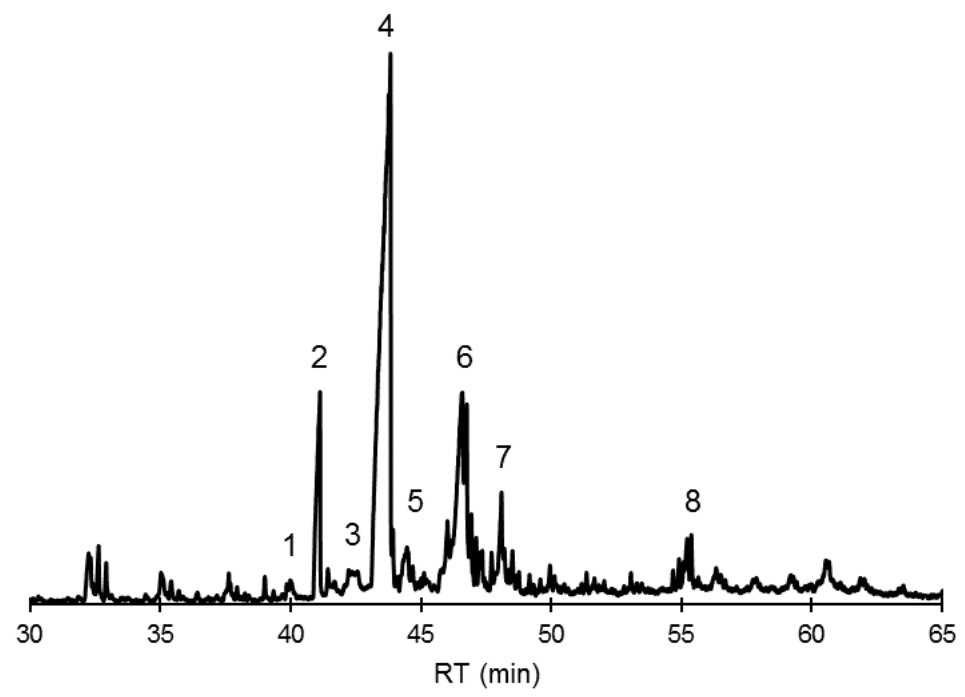

Figure S4: GC-MS chromatogram of $\mathrm{P} 3\left(\mathrm{GC}-\mathrm{MS}\right.$ : $\left.\mathrm{Ti}=35^{\circ} \mathrm{C}, \mathrm{HR}=3^{\circ} \mathrm{C} / \mathrm{min}\right)$.

Table S4: chemical structures of P3 identified using GC-MS.

Peak
number




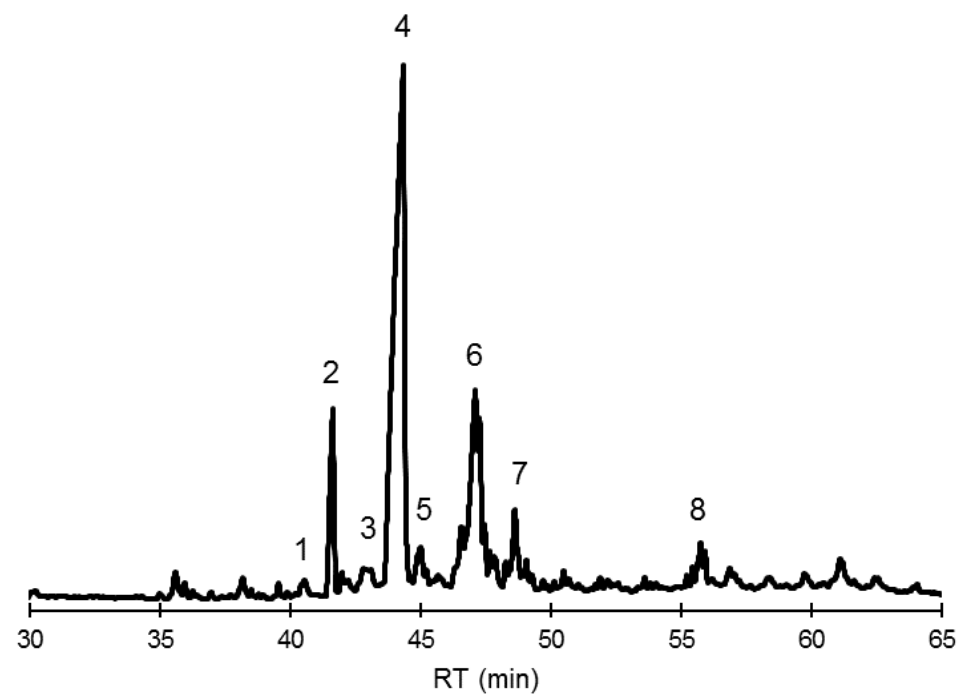

Figure S5: GC-MS chromatogram of $\mathrm{P} 4\left(\mathrm{GC}-\mathrm{MS}\right.$ : $\left.\mathrm{Ti}=35^{\circ} \mathrm{C}, \mathrm{HR}=3^{\circ} \mathrm{C} / \mathrm{min}\right)$.

Table S5: chemical structures of P4 identified using GC-MS.

Peak
number




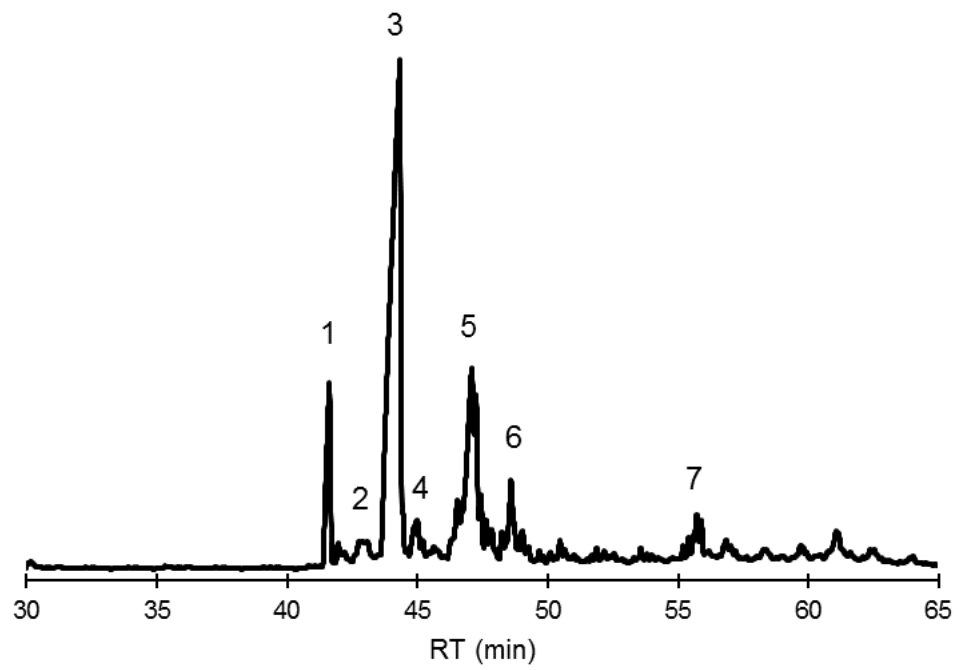

Figure $\mathrm{S6}$ : GC-MS chromatogram of $\mathrm{P} 5\left(\mathrm{GC}-\mathrm{MS}\right.$ : $\left.\mathrm{Ti}=35^{\circ} \mathrm{C}, \mathrm{HR}=3^{\circ} \mathrm{C} / \mathrm{min}\right)$.

Table S6: chemical structures of P5 identified using GC-MS.

Peak
number




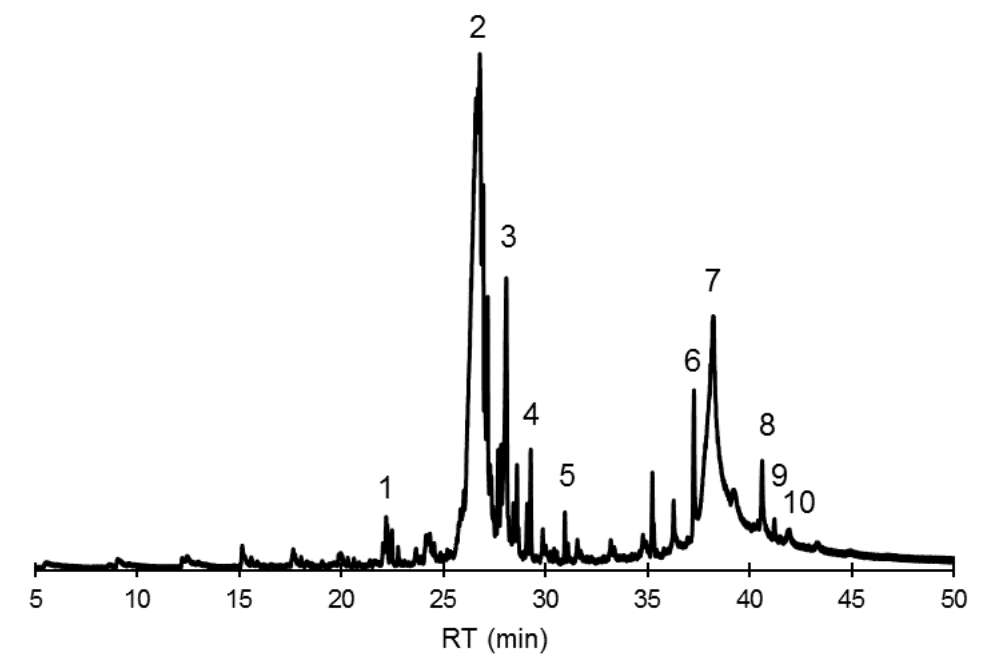

Figure S7: GC-MS chromatogram of P6.

Table S7: chemical structures of P6 identified using GC-MS.

(n)




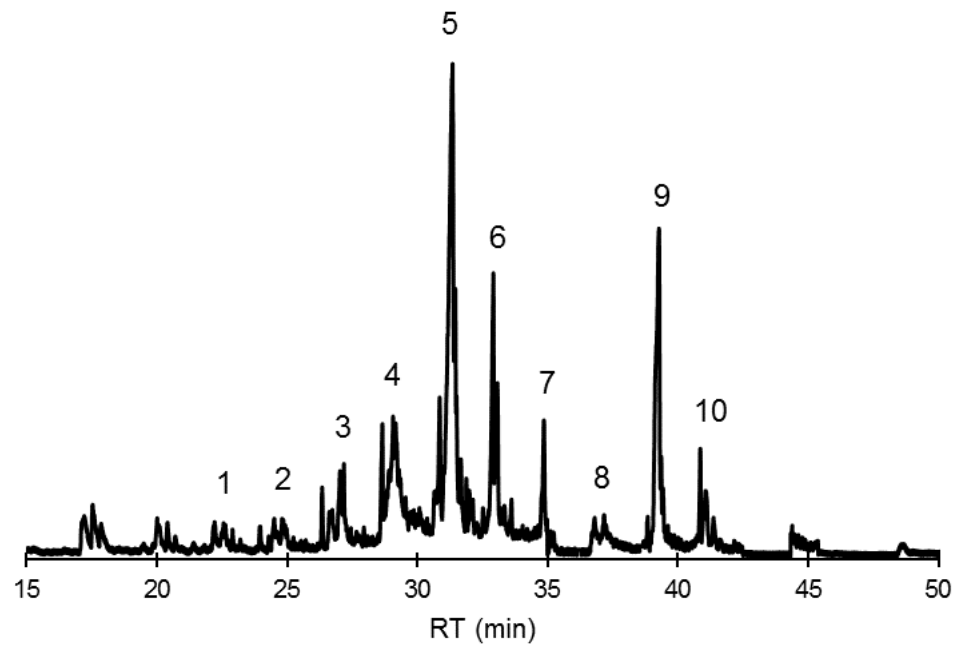

Figure S8: GC-MS chromatogram of P7.

Table S8: chemical structures of P7 identified using GC-MS.

Peak
number




9




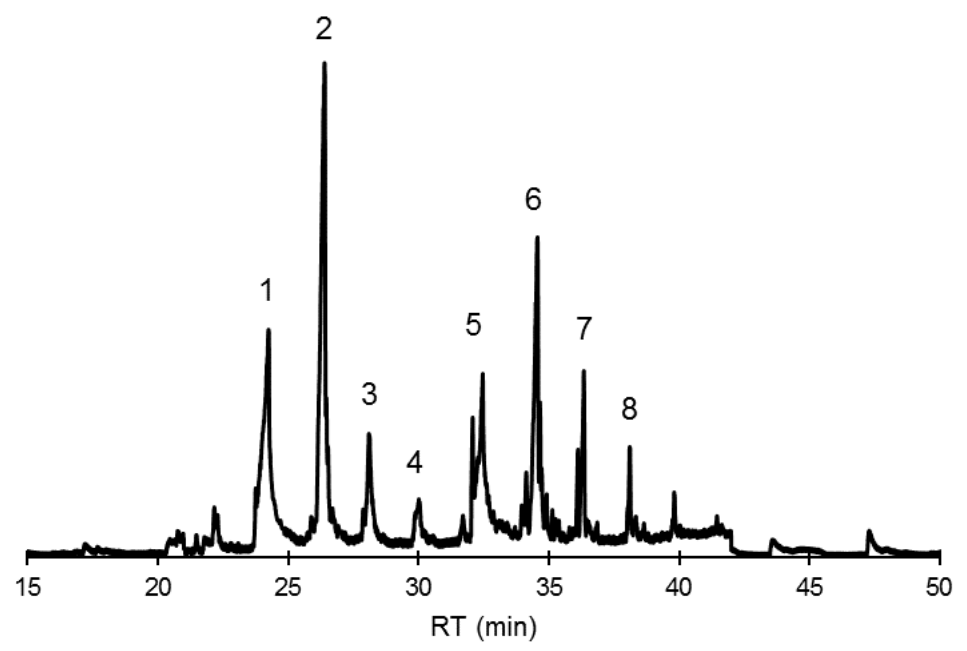

Figure S9: GC-MS chromatogram of P8.

Table S9: chemical structures of P7 identified using GC-MS.

Peak
number




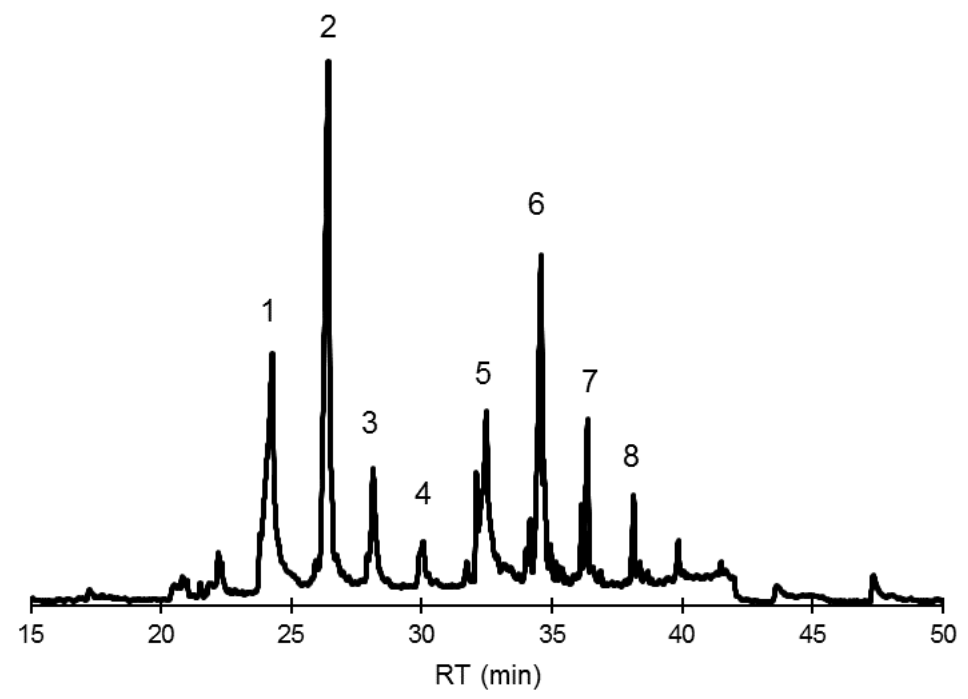

Figure S10: GC-MS chromatogram of P9.

Table S10: chemical structures of P9 identified using GC-MS.

\begin{tabular}{|c|c|}
\hline $\begin{array}{l}\text { Peak } \\
\text { number }\end{array}$ & Identified chemical structure \\
\hline 1 & \\
\hline 2 & \\
\hline 3 & \\
\hline 4 & \\
\hline 5 & \\
\hline 6 & \\
\hline 7 & \\
\hline 8 & \\
\hline
\end{tabular}




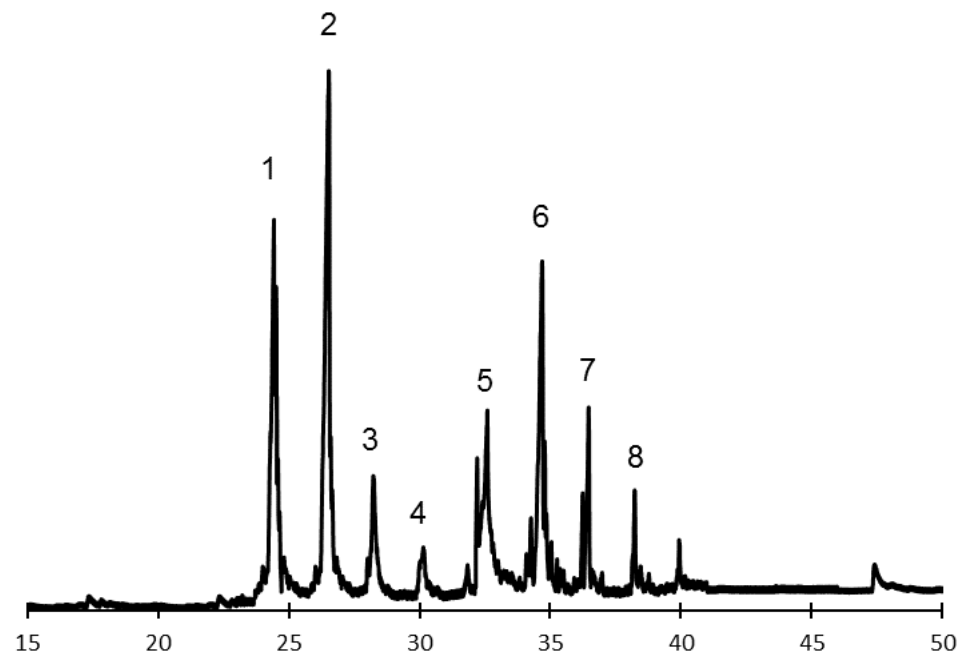

Figure S11: GC-MS chromatogram of P10.

Table S11: chemical structures of P10 identified using GC-MS.

Peak




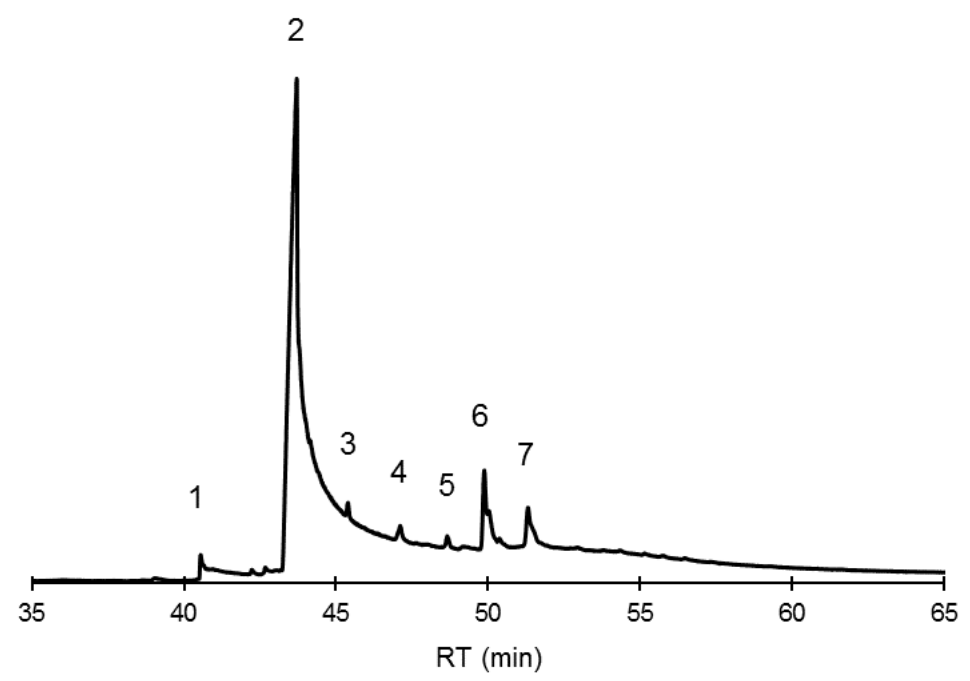

Figure S12: GC-MS chromatogram of P11 (GC-MS: $\left.\mathrm{Ti}=35^{\circ} \mathrm{C}, \mathrm{HR}=3^{\circ} \mathrm{C} / \mathrm{min}\right)$.

Table S12: chemical structures of P11 identified using GC-MS.

Peak




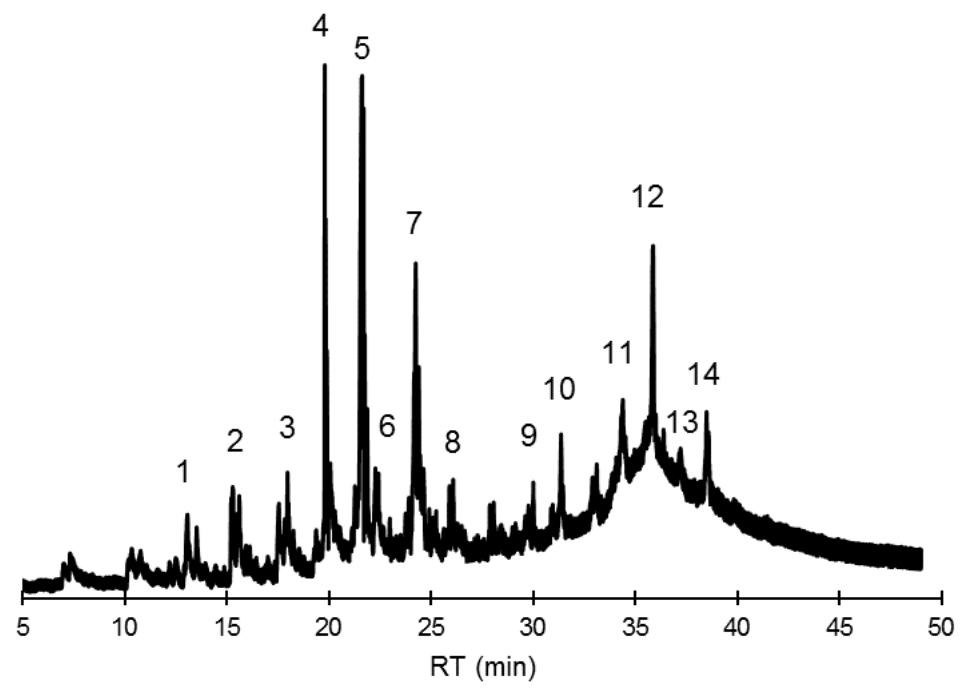

Figure S13: GC-MS chromatogram of $\mathrm{P} 12\left(\mathrm{GC}-\mathrm{MS}\right.$ : $\left.\mathrm{Ti}=40^{\circ} \mathrm{C}, \mathrm{HR}=5^{\circ} \mathrm{C} / \mathrm{min}\right)$.

Table S13: chemical structures of P12 identified using GC-MS.

(ldentified chemical structure



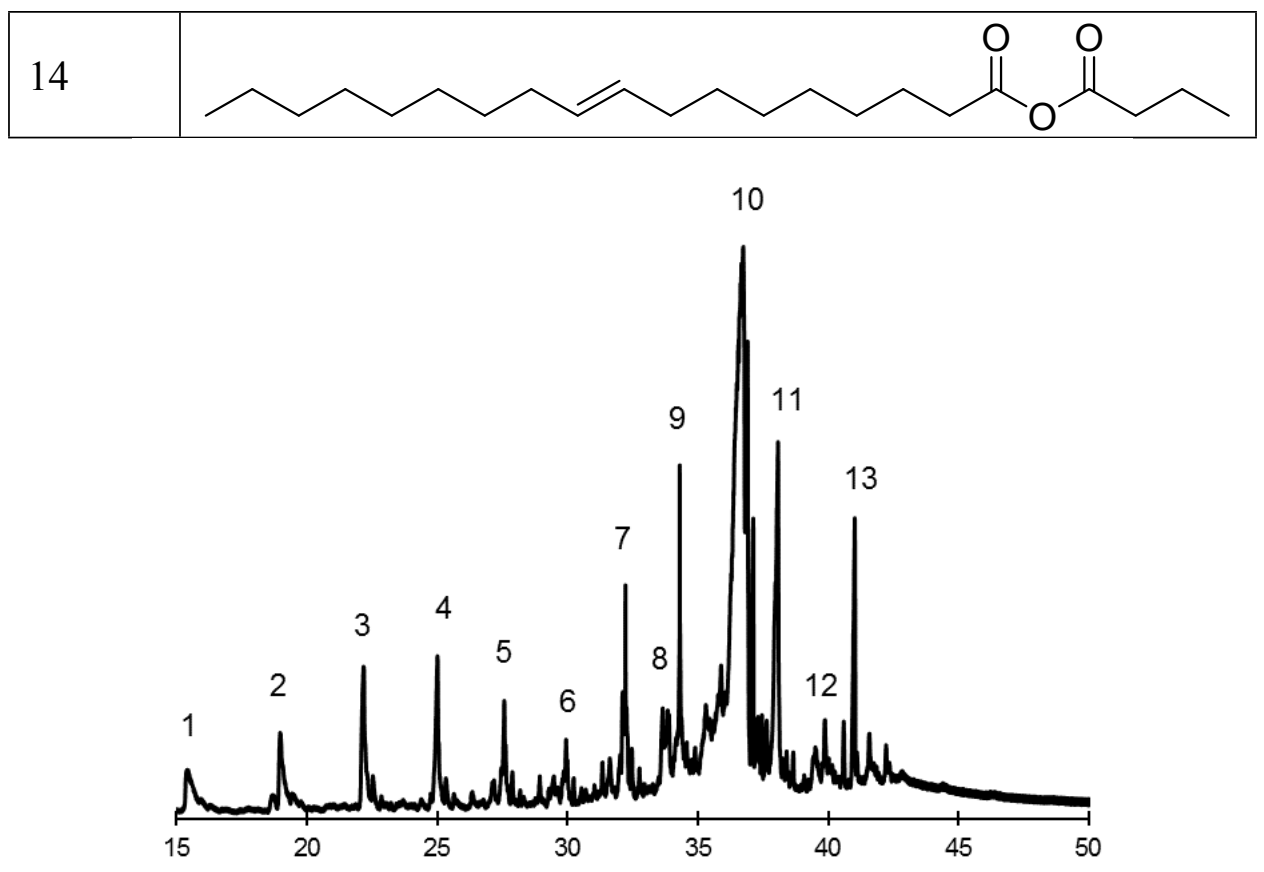

Figure S14: GC-MS chromatogram of $\mathrm{P} 13\left(\mathrm{GC}-\mathrm{MS}\right.$ : $\left.\mathrm{Ti}=40^{\circ} \mathrm{C}, \mathrm{HR}=5^{\circ} \mathrm{C} / \mathrm{min}\right)$.

Table S14: chemical structures of P13 identified using GC-MS.

Pumber




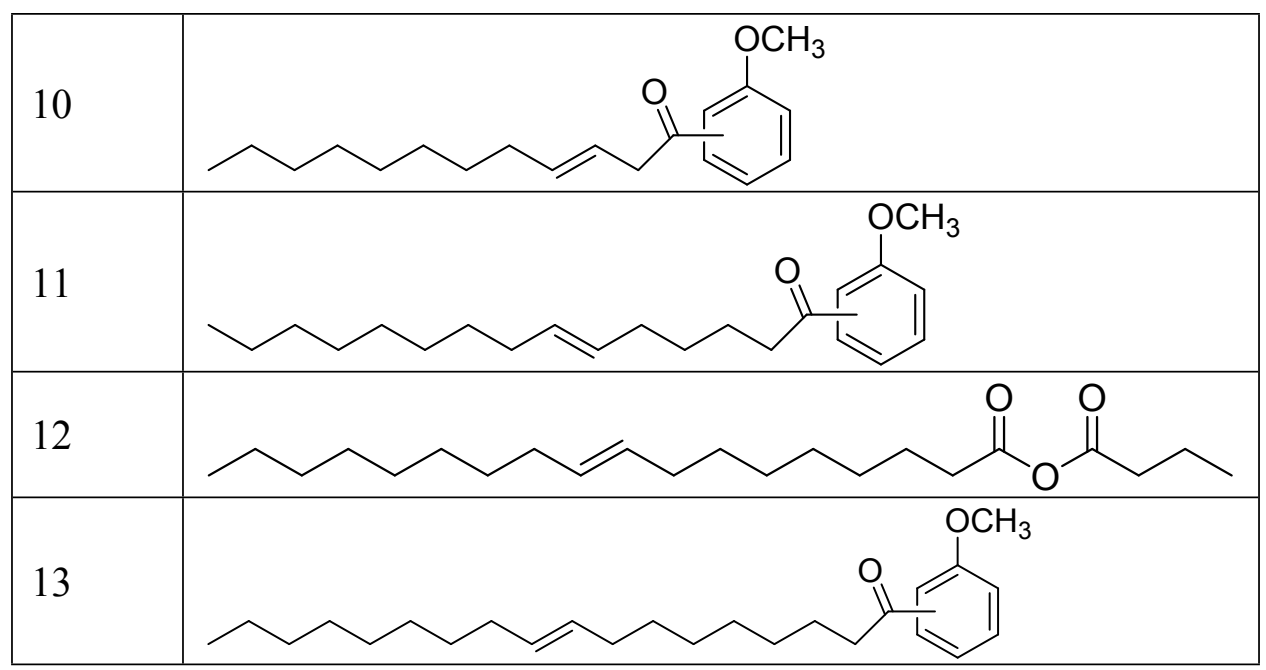




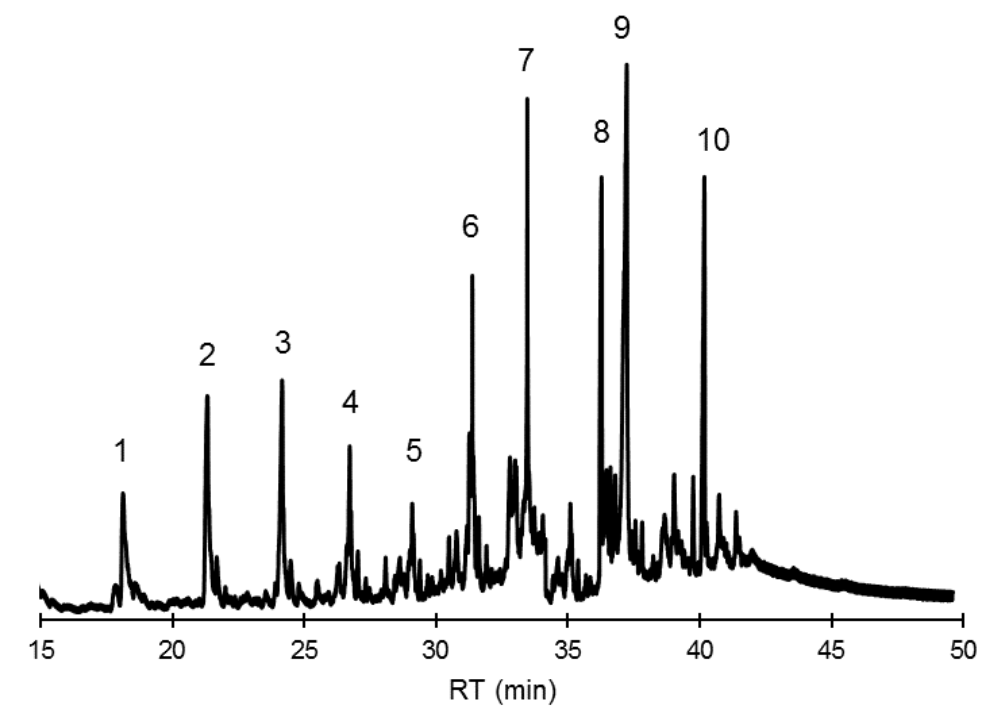

Figure S15: GC-MS chromatogram of P14.

Table S15: chemical structures of P14 identified using GC-MS.

Peak
number




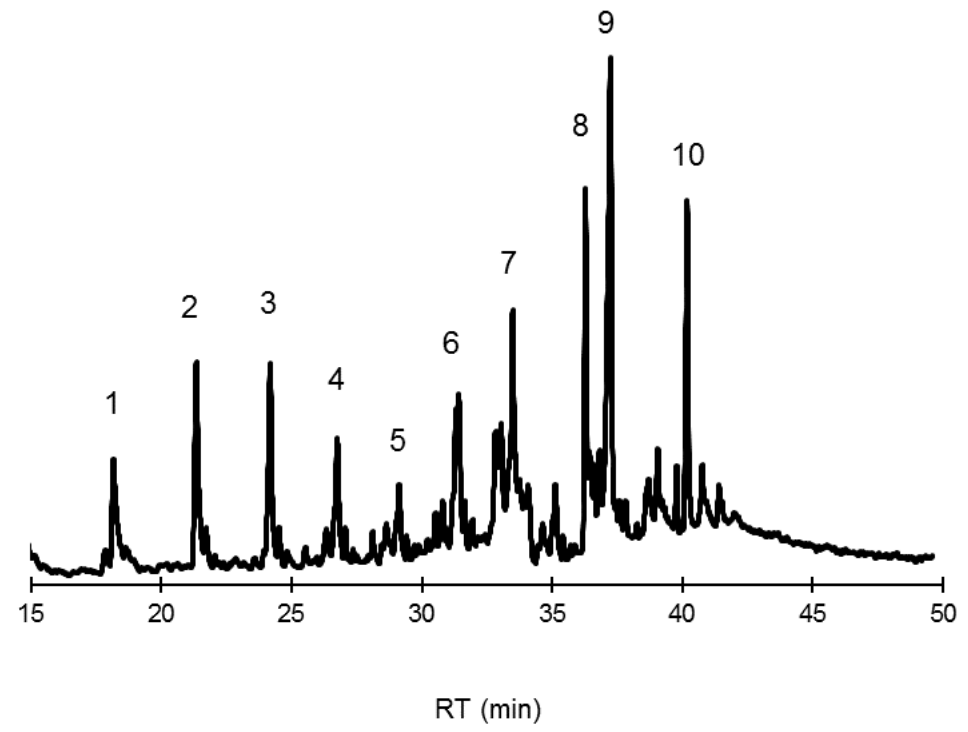

Figure S16: GC-MS chromatogram of P15.

Table S16: chemical structures of P15 identified using GC-MS.

\begin{tabular}{|c|c|}
\hline $\begin{array}{l}\text { Peak } \\
\text { number }\end{array}$ & Identified chemical structure \\
\hline 1 & $\leadsto / \sim$ \\
\hline 2 & \\
\hline 3 & $\widehat{\Upsilon}$ \\
\hline 4 & $\wedge$ \\
\hline 5 & 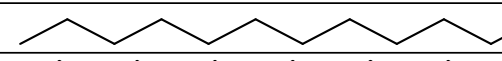 \\
\hline 6 & \\
\hline 7 & \\
\hline 8 & 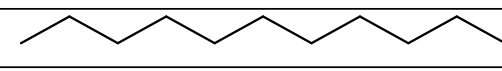 \\
\hline 9 & \\
\hline 10 & \\
\hline
\end{tabular}




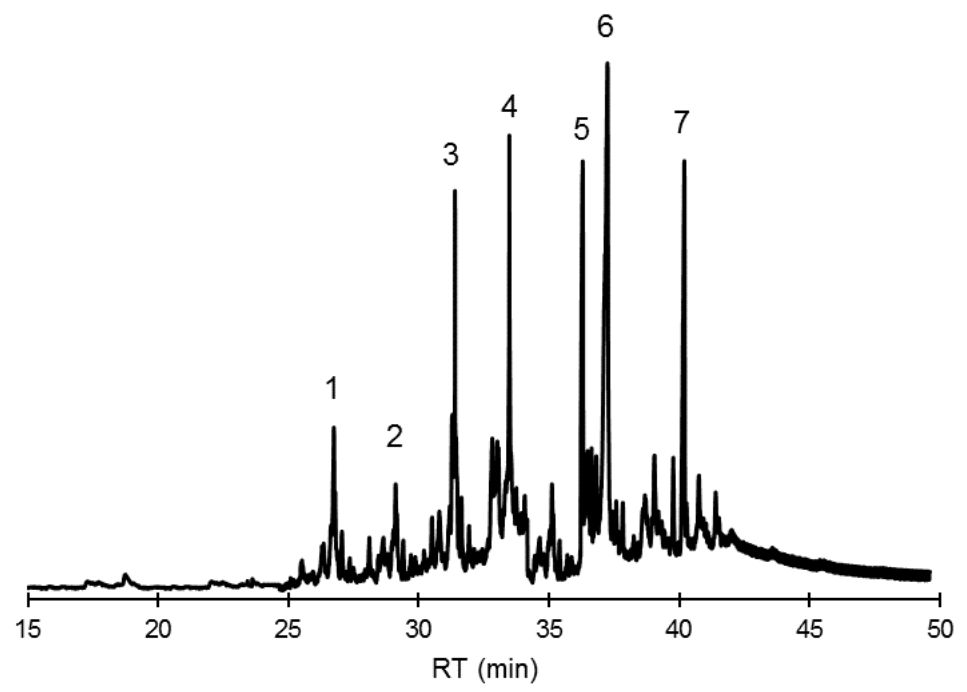

Figure S17: GC-MS chromatogram of P16.

Table S17: chemical structures of P16 identified using GC-MS.

\begin{tabular}{|c|c|}
\hline $\begin{array}{l}\text { Peak } \\
\text { number }\end{array}$ & Identified chemical structure \\
\hline 1 & \\
\hline 2 & \\
\hline 3 & \\
\hline 4 & \\
\hline 5 & \\
\hline 6 & \\
\hline 7 & \\
\hline
\end{tabular}




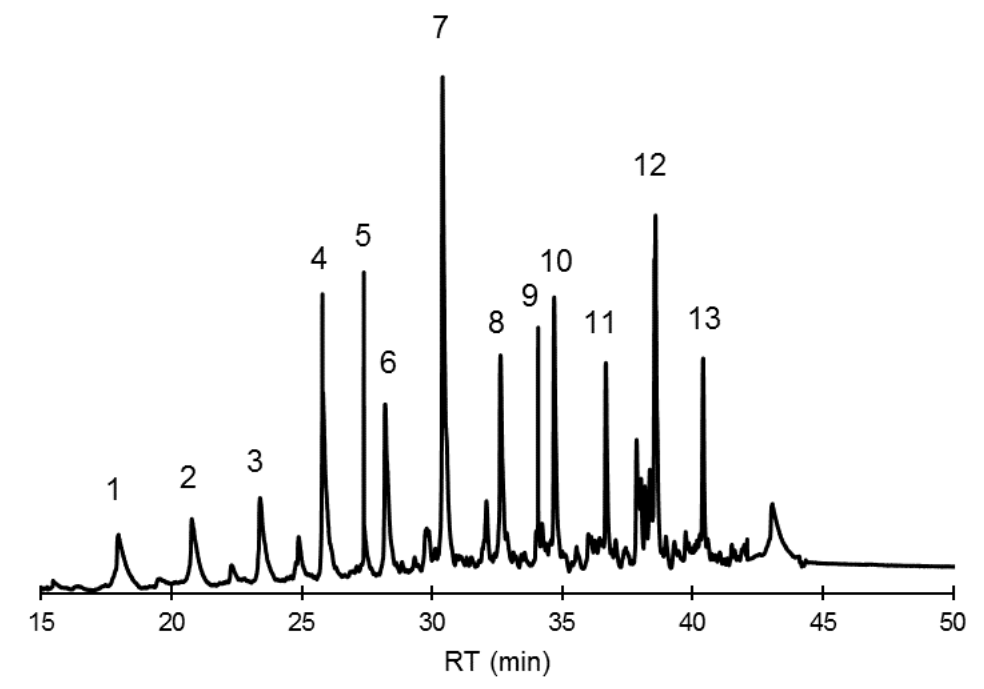

Figure S18: GC-MS chromatogram of P17.

Table S18: chemical structures of P17 identified using GC-MS (GC-MS: $\mathrm{Ti}=60^{\circ} \mathrm{C}$, $\left.\mathrm{HR}=5^{\circ} \mathrm{C} / \mathrm{min}\right)$.

(Peak



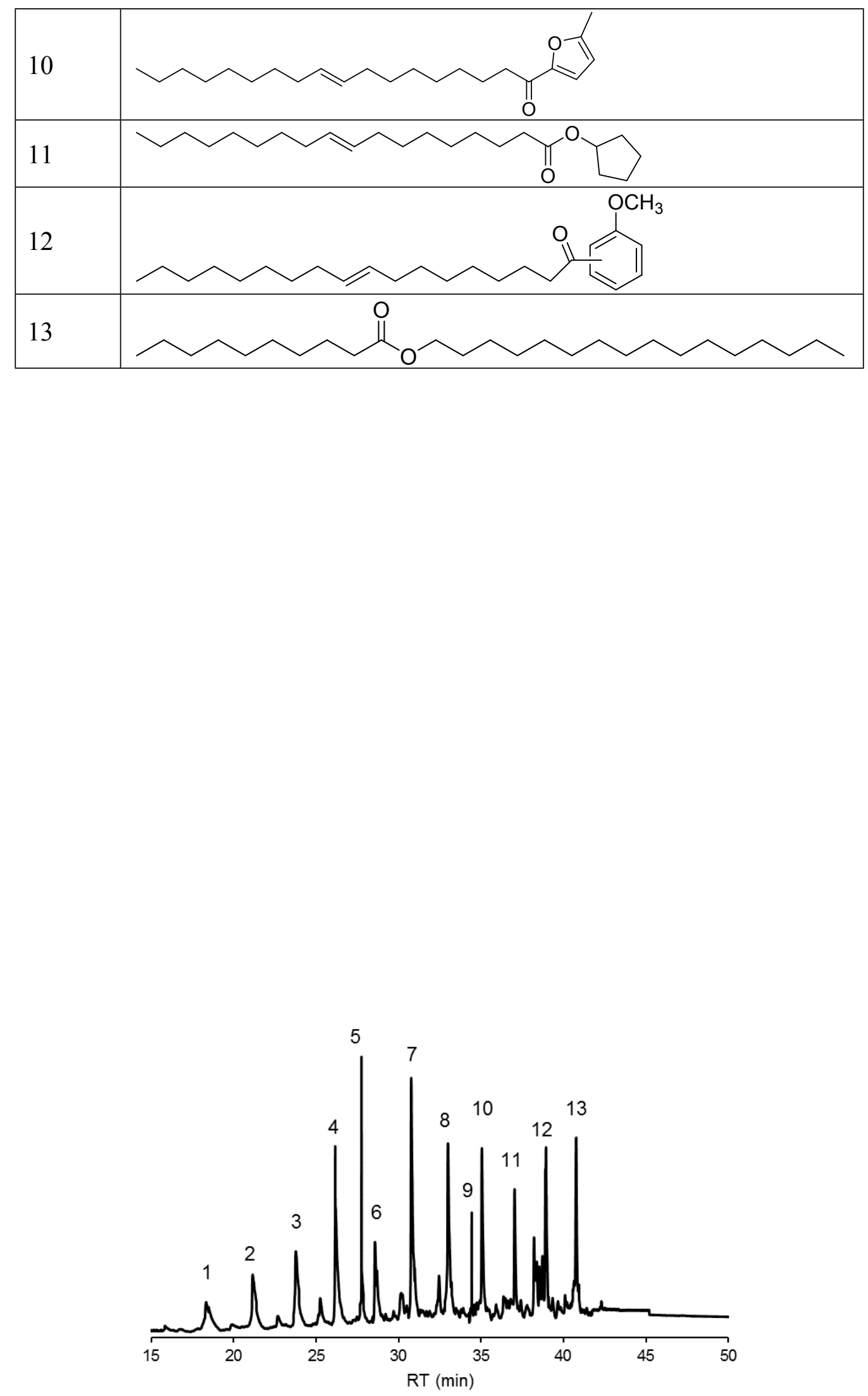
Figure S19: GC-MS chromatogram of P18.

Table S19: chemical structures of P18 identified using GC-MS (GC-MS: $\mathrm{Ti}=60^{\circ} \mathrm{C}$, $\left.\mathrm{HR}=5^{\circ} \mathrm{C} / \mathrm{min}\right)$.

(1)

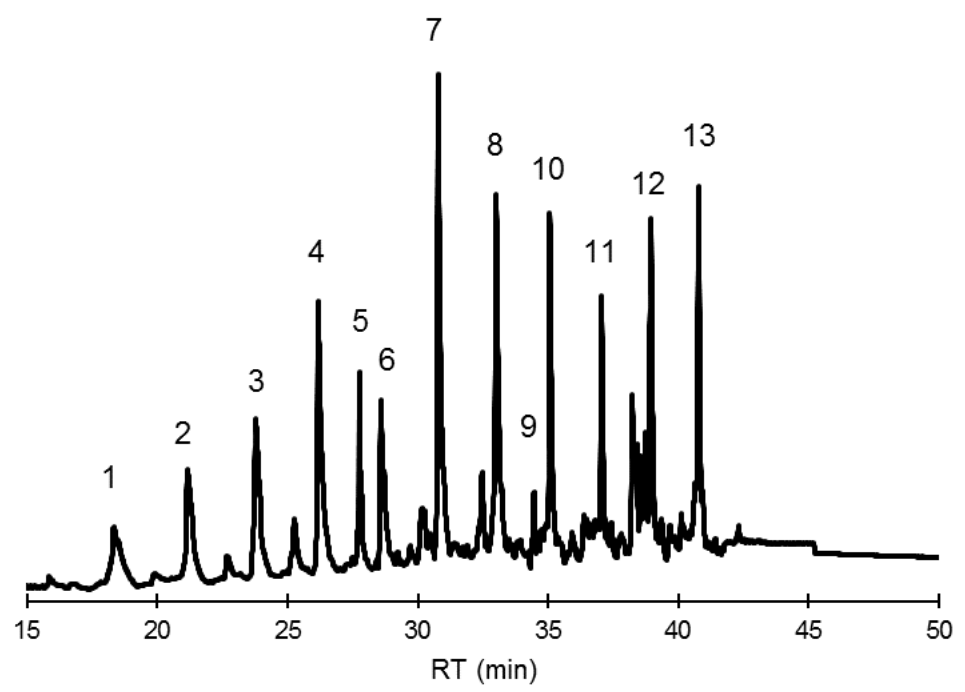


Figure S20: GC-MS chromatogram of $\mathrm{P} 19\left(\mathrm{GC}-\mathrm{MS}\right.$ : $\left.\mathrm{Ti}=60^{\circ} \mathrm{C}, \mathrm{HR}=5^{\circ} \mathrm{C} / \mathrm{min}\right)$.

Table S20: chemical structures of P19 identified using GC-MS.

(1)

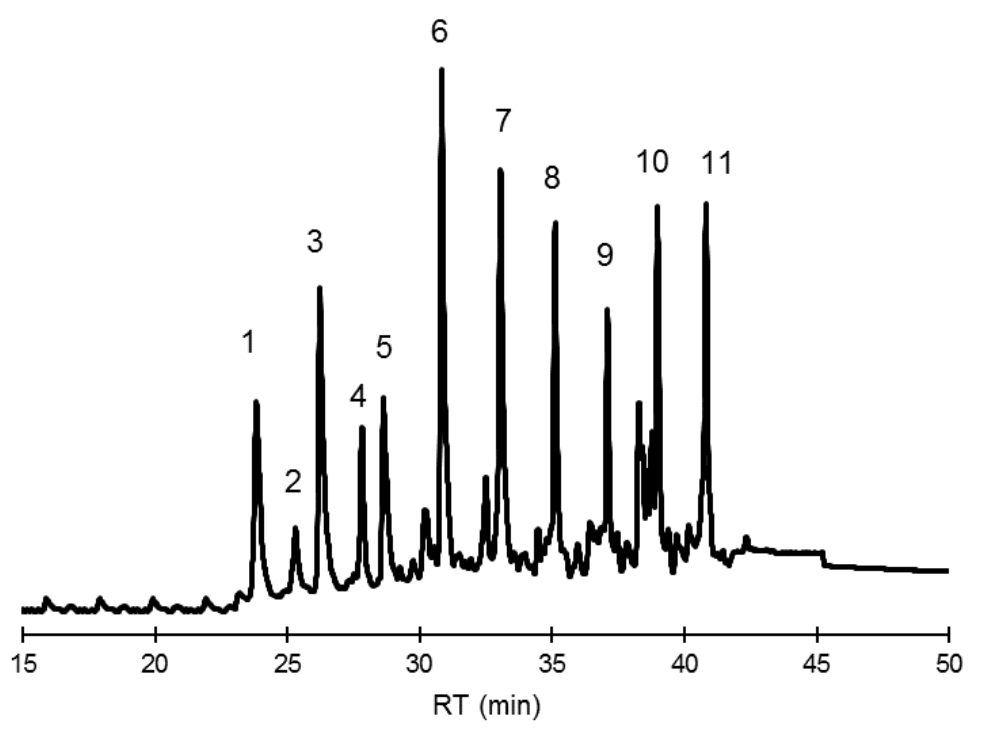


Figure S21: GC-MS chromatogram of $\mathrm{P} 20\left(\mathrm{GC}-\mathrm{MS}\right.$ : $\left.\mathrm{Ti}=60^{\circ} \mathrm{C}, \mathrm{HR}=5^{\circ} \mathrm{C} / \mathrm{min}\right)$.

Table S21: chemical structures of P20 identified using GC-MS.

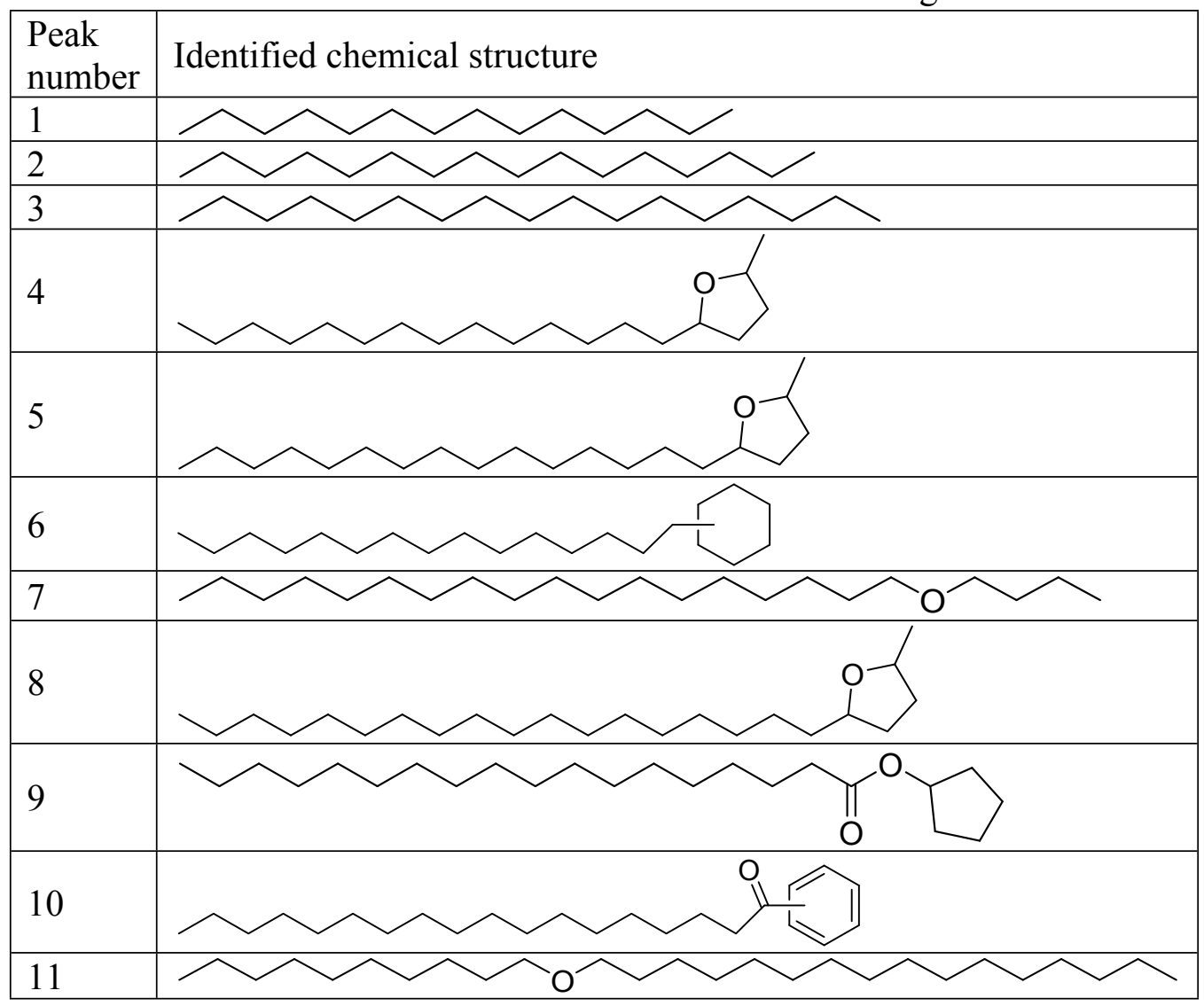

